\title{
Broadband optical ultrasound sensor with a unique open-cavity structure
}

\author{
Colin M. Chow, ${ }^{\mathrm{a}}$ Yun Zhou, ${ }^{\mathrm{b}}$ Yunbo Guo, ${ }^{\mathrm{a}}$ Theodore B. Norris, ${ }^{\mathrm{a}}$ Xueding Wang, ${ }^{\mathrm{c}}$ Cheri X. Deng, ${ }^{\mathrm{b}}$ and Jing Yong Ye ${ }^{\mathrm{d}, *}$ \\ a University of Michigan, Department of Electrical Engineering and Computer Science, Ann Arbor, Michigan 48109 \\ buniversity of Michigan, Department of Biomedical Engineering, Ann Arbor, Michigan 48109 \\ 'University of Michigan, Department of Radiology, Ann Arbor, Michigan 48109 \\ ${ }^{\mathrm{d} U n i v e r s i t y ~ o f ~ T e x a s ~ a t ~ S a n ~ A n t o n i o, ~ D e p a r t m e n t ~ o f ~ B i o m e d i c a l ~ E n g i n e e r i n g, ~ S a n ~ A n t o n i o, ~ T e x a s ~} 78249$
}

\begin{abstract}
High-resolution ultrasound imaging requires quality sensors with wide bandwidth and high sensitivity, as shown in a wide range of applications, including intravascular imaging of cardiovascular diseases. However, piezoelectric technology, the current dominant approach for hydrophone fabrication, has encountered many technical limitations in the high-frequency range. Using optical techniques for the detection of high-frequency ultrasound signals has attracted much recent attention. One of the most studied approaches is based on a Fabry-Pérot interferometer, consisting of an optical cavity sandwiched between two mirrors. This technique offers promising sensitivity and bandwidth, and a potential alternative to piezoelectric polyvinylidene fluoride (PVDF) hydrophones. We propose an innovative optical ultrasound sensor using only a single mirror in a total-internal-reflection configuration. Besides retaining the advantages of Fabry-Pérot interferometer-based ultrasound sensors, this unique design provides a bandwidth of at least $160 \mathrm{MHz}$, a potential decrease in fabrication cost, and an increase in signal fidelity. (C) 2011 Society of Photo-Optical Instrumentation Engineers (SPIE). [DOI: 10.1117/1.3528014]
\end{abstract}

Keywords: optical ultrasound sensor; ultrasound imaging; photoacoustic imaging; hydrophone.

Paper 10077RRRR received Feb. 16, 2010; revised manuscript received Nov. 2, 2010; accepted for publication Nov. 8, 2010; published online Jan. 18, 2011.

There is a pervasive demand for high-resolution imaging techniques for comprehensive morphological and functional imaging of critical biological organs covered by thick tissue layers. Optical microscopy normally provides superior resolution but with shallow imaging depth in scattering media. In contrast, acoustic imaging enables deep tissue imaging but with relatively low spatial resolution, restricted by the frequency range employed in conventional diagnostic ultrasound imaging. In principle, ultrasound imaging resolution can be improved by using higher frequencies (e.g., $50 \mathrm{MHz}$ ). However, for a conventional piezoelectric-based ultrasound sensor, higher operation frequency demands smaller effective sensor size,${ }^{1}$ which imposes several technological difficulties in the fabrication of miniature piezoelectric ultrasound sensors. The challenges include dicing piezoceramics to micrometer-size elements, ${ }^{2}$ making electrical connections to the small elements, cross talk between elements, and increased detection noise due to reduced element size. ${ }^{3}$ Although recently developed miniature polyvinylidene fluoride (PVDF) membrane hydrophones can potentially achieve $\mathrm{e}^{4,5}$ a frequency bandwidth beyond $140 \mathrm{MHz}$, demanding electrical connections and cross talk persist if these membrane hydrophones are intended for imaging arrays. Furthermore, it has been reported that the noise-equivalent pressure (NEP) of a 50- $\mu$ m-diam Fabry-Pérot optical ultrasound sensor is comparable to that of 1-mm-diam PVDF membrane hydrophone. ${ }^{6}$ Given that the sensitivity of a membrane hydrophone decreases as its area becomes smaller due to lower capacitance, ${ }^{7}$ the Fabry-Pérot ultrasound sensor can potentially

Address all correspondence to: Jing Yong Ye, University of Texas at San Antonio, Biomedical Engineering, One UTSA Circle, San Antonio, TX 78249. Tel: 210458-5056; E-mail: jingyong.ye@utsa.edu. outperform the PVDF membrane sensor with the same active area. $^{8}$

As discussed in recent publications, , $^{3,6-16}$ optical ultrasound sensors have the potential to overcome many of the challenges just mentioned. For example, no electrical connections are required near an optical ultrasound sensor and, in most of the optical techniques employed, micrometer-size resolution can be obtained by scanning a focused laser beam across sensor surface. ${ }^{9}$ A Fabry-Pérot étalon is one of the well-studied structures $^{3,7,10-16}$ for building such an optical ultrasound sensor due to its superior frequency response and sensitivity relative to conventional piezoelectric equivalents. ${ }^{6}$ The operation of a Fabry-Pérot étalon ultrasound sensor is based on modulation of optical cavity length by ultrasonic pressure wave. As reported by Zhang et al., ${ }^{8}$ the fabrication of a high-finesse étalon ultrasound sensor can be achieved by first sputtering a stack of two alternating periodic dielectric layers, also called a photonic crystal (PC) structure, on a transparent substrate to form a high-reflection mirror, then the cavity material is deposited, followed by sputtering the second mirror. A broad bandwidth of $20 \mathrm{MHz}$ was achieved with a sensor having a cavity layer of 38 or $22 \mu \mathrm{m}$. In addition, the sensitivity of the étalon-based sensors was speculated to be potentially two orders of magnitude more sensitive than a piezoelectric PVDF receiver with similar element size. ${ }^{8}$

Two important parameters determining the performance of the étalon-based optical ultrasound sensor are the finesse of the étalon and the Young's modulus of the cavity material. Onedimensional PC structures can be used to fabricate an étalon sensor with high finesse by sputtering dielectric multilayers on both sides of a cavity layer. For coating with hard dielectric

1083-3668/2011/16(1)/017001/6/\$25.00 (C) 2011 SPIE 
(a)

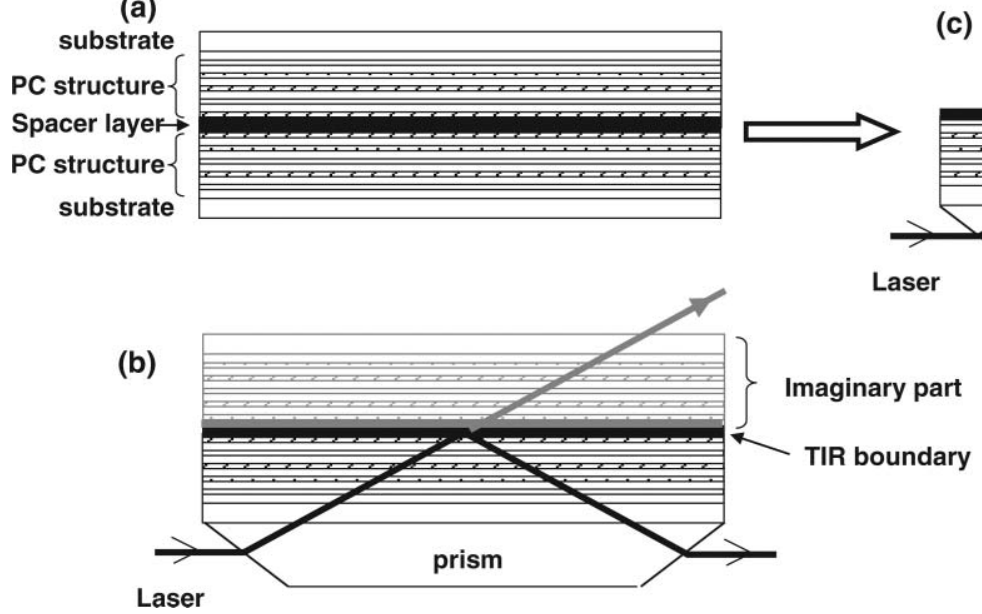

(c) Ultrasound

signal

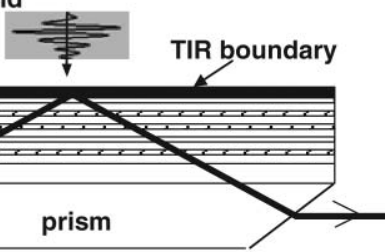

Fig. 1 Working principle of a sensor with an open-cavity structure: (a) the conceptual split of an étalon sensor having a cavity layer sandwiched by two PC structures from the middle of the cavity layer; (b) only half of the structure is used in TIR geometry, owing to the TIR, a mirror image of the PC structure results in a virtual étalon structure; and (c) a PC-TIR optoacoustic sensor with an open cavity, which ultimately enables optimizing the ultrasound detection sensitivity and bandwidth.

materials, including $\mathrm{SiO}_{2}$ and $\mathrm{TiO}_{2}$, the cavity material must meet several requirements to ensure good coating quality: $(i)$ sufficient rigidity, (ii) a high melting point, and (iii) a thermal expansion coefficient matched with the dielectric multilayers. Yet such materials have intrinsically limited response to an ultrasound signal. For example, an étalon sensor with fused silica as the cavity layer was fabricated, but the detection sensitivity is limited ${ }^{7}$ due to silica's high Young's modulus, which limits the change in cavity length induced by ultrasonic pressure waves. Polymer films with lower Young's moduli were used in an attempt to increase the sensitivity. ${ }^{3,14}$ However, it is difficult to obtain a high-quality coating with hard dielectric materials on most polymer films due to the preceding strict requirements on substrate temperature and rigidity. A soft dielectric coating can be used instead, which lowers the requirements on the substrate physical properties, but the coating is less robust than a hard dielectric coating. Optical ultrasound sensors with parylene $\mathrm{C}$ as the cavity layer, which has a melting point of $290^{\circ} \mathrm{C}$ and Young's modulus of $4.75 \mathrm{GPa},{ }^{17}$ was successfully fabricated using soft dielectric coating of $\mathrm{ZnS}$ and $\mathrm{Na}_{3} \mathrm{AlF}_{6}$ on a polymethyl-methacrylate (PMMA) substrate. ${ }^{8}$ Instead of coating dielectric materials on a polymer film, metal coating was normally used, ${ }^{3,14}$ which however resulted in a lower finesse due to the relatively low reflectivity. Thus, the detection sensitivity of the polymer étalon sensor is not optimized either. To overcome these limitations due to the trade-off between the finesse of the étalon structure and the compressibility of the cavity layer material, in this paper we demonstrate an alternative approach for constructing a high-frequency optical ultrasound sensor with an open-cavity configuration.

We conceptually split a Fabry-Pérot étalon sensor with a cavity layer sandwiched between two PC structures from the middle and use only half of the structure in a total-internalreflection (TIR) geometry [Fig. 1(a)]. Owing to the TIR, there is a mirror image of the PC structure [Fig. 1(b)]. Thus, a cavity is still formed even with only half of a conventional étalon structure due to the PC structure and its imaginary part in the TIR configuration. Note that this is an open cavity [Fig. 1(c)], in contrast to the closed cavity in a conventional étalon sensor.
The existence of resonant modes similar to that of a FabryPérot étalon cavity has been verified in our previous experiments utilizing the open cavity for real-time bioassays. ${ }^{18,19}$ This PCTIR sensor (a PC structure used in TIR geometry) has a number of advantages. First, a variety of materials can be used for the cavity layer, because no coating is necessary on top of the cavity layer. Thus, the selection of materials fully depends on their capability for achieving a large optical response to broadband high-frequency ultrasonic signals rather than having to meet the requirements of fabrication processes. Second, comparing to a sandwiched étalon structure, the open-cavity configuration also enables the sensing layer to be more flexible, because not both sides of the layer are coated with dielectric materials that could increase the cavity mechanical toughness and reduce the sensor response. Finally, the PC-TIR sensor retains most of the advantages of other Fabry-Pérot ultrasound sensors. However, because of the required oblique incident angle of the probe beam, the spatial resolution of the PC-TIR sensor is degraded by a factor of 1 over cosine of the incident angle compared to a Fabry-Pérot ultrasound sensor with the normal incidence of a probe beam having the same beam diameter.

We designed the PC-TIR sensor based on the following theoretical calculations. The sensor is composed of a transparent substrate, a periodic structure of two alternating layers of different dielectric materials (e.g., silica and titania), and a polymer thin film as the cavity layer. Assume the incident angle at the substrate layer is $\theta_{i}$, and the refraction angles in the substrate (S), silica (A), titania (B), and cavity layer (X) are $\theta_{s}, \theta_{A}, \theta_{B}$, and $\theta_{X}$, respectively. Let $n_{s}, n_{A}, n_{B}$, and $n_{X}$ be the refractive indices of the substrate, silica, titania, and the cavity layer, respectively. To form a photonic bandgap, the thickness of the dielectric multilayer must satisfy

$d_{\mathrm{A}}=\frac{\lambda}{4\left(n_{\mathrm{A}}^{2}-n_{\mathrm{S}}^{2} \sin ^{2} \theta_{\mathrm{S}}\right)^{1 / 2}}, \quad d_{\mathrm{B}}=\frac{\lambda}{4\left(n_{\mathrm{B}}^{2}-n_{\mathrm{S}}^{2} \sin ^{2} \theta_{\mathrm{S}}\right)^{1 / 2}}$,

where $d_{A}$ and $d_{B}$ are the thicknesses for silica and titania, respectively and $\lambda$ is the wavelength of the probe laser, which was 

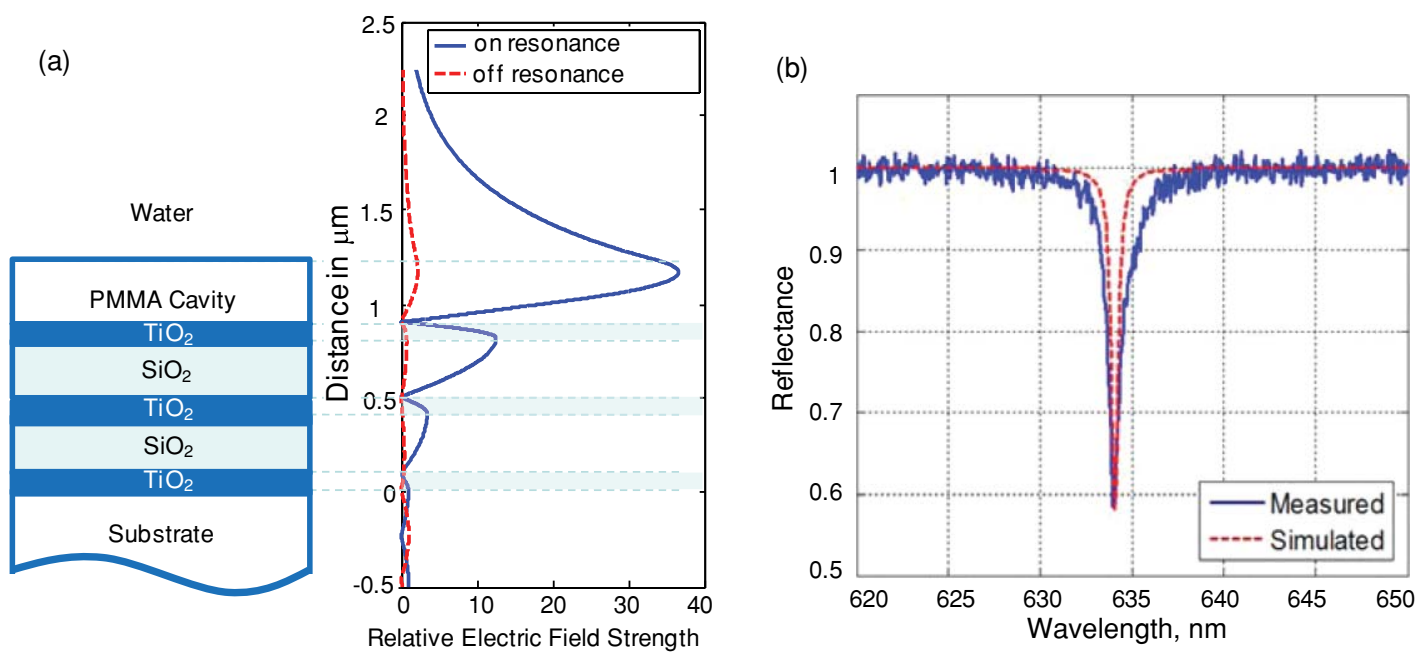

Fig. 2 Resonance condition of the PC-TIR sensor. (a) Electric field profiles in a PC-TIR sensor at an incident angle of 64 deg simulated with a transfer matrix method. The dashed line shows the case without resonance for compressed cavity thickness of $0.99 d_{x}$, while the solid line shows the case with resonance at $d_{x}=0.334 \mu \mathrm{m}$. The strong field enhancement in the PMMA layer indicates the existence of a cavity in this open structure. (b) Simulated and measured reflectance spectra for a PC-TIR sensor with a PMMA cavity thickness of $2.65 \mu \mathrm{m}$. The measured reflectance dip is as narrow as $1 \mathrm{~nm}$, which is in a good agreement with the simulated result.

chosen to be $632.8 \mathrm{~nm}$ in this experiment. An incident angle of 64 deg was chosen, so that the TIR occurs at the polymer-water interface but not at the titania-silica interface. At this incident angle, the titania and silica layers therefore have designed thicknesses of 89.8 and $307.2 \mathrm{~nm}$, respectively. They were used to form five alternating layers of the PC structure and were fabricated by electron-beam physical vapor deposition on a BK7 glass substrate.

To obtain a resonance condition in the cavity, the thickness of the cavity layer $d_{x}$ should satisfy the following relation:

$$
2 \frac{2 \pi}{\lambda_{\mathrm{d}}} n_{x} d_{x} \cos \theta_{x}+\alpha=(2 m+1) \pi \quad(m=0,1,2, \ldots),
$$

where $\alpha$ represents the Goos-Hänchen phase shift. The factor of 2 in the first term on the left-hand side is due to the fact that the light double passes this layer because of the TIR. The integer $m$ means that multiple resonant conditions can be satisfied. For $s$-polarization of incident light, substituting the Goos-Hänchen phase shift expression into Eq. (2), one obtains the thickness of the cavity layer,

$$
\begin{aligned}
d_{X}= & \frac{\lambda_{\mathrm{d}}}{4 \pi\left(n_{\mathrm{X}}^{2}-n_{\mathrm{S}}^{2} \sin ^{2} \theta_{\mathrm{S}}\right)^{1 / 2}} \\
& \times\left\{2 m \pi+\pi-2 \tan ^{-1}\left[\left(\frac{n_{\mathrm{S}}^{2} \sin ^{2} \theta_{\mathrm{S}}-n_{\mathrm{H}}^{2}}{n_{\mathrm{X}}^{2}-n_{\mathrm{S}}^{2} \sin ^{2} \theta_{\mathrm{S}}}\right)^{1 / 2}\right]\right\},
\end{aligned}
$$

where $n_{H}$ is the refractive index of the medium above the cavity layer.

We chose PMMA as the material for the cavity layer due to its good elasticity property for achieving high sensitivity and the ease of depositing PMMA by spin-coating. PMMA has a low Young's modulus of $2 \mathrm{GPa}$ as compared to titania (230 GPa) and silica (72 GPa). The PC-TIR sensor is essentially an interferometer with its optical resonant wavelengths determined by the thickness of the PMMA layer. When exposed to an ultrasound wave, the ultrasonic pressure wave modulates the thickness of the PMMA cavity, causing it to be on and off resonance. While off resonance, the optical electric field within the cavity layer destructively interferes and the field is minimal [Fig. 2(a)]. Upon resonance, the probe light is confined within the cavity due to constructive interference, and field enhancement occurs. However, because no light is transmitted to the other side of cavity layer in TIR, conservation of energy requires the reflectance of the interferometer to be unity regardless of whether the cavity is on or off resonance. To distinguish these two cases, a small amount of light-absorbing dye (50 ppm of Nile blue dye) is incorporated into the PMMA cavity layer. Because of the field enhancement in this layer under the resonant condition, the maximum absorption occurs and the reflectance spectrum shows a significant dip at the resonant wavelength [Fig. 2(b)].

The reason why it is necessary to dope a small amount of absorbing materials in the cavity layer can be understood in the following way. If there was no absorption at all in the cavity layer, then the reflectivity would remain high (basically, close to unity) across the whole spectrum and there would be no resonant dip. This is because for light that is not at the resonant wavelength, it has minimal field intensity due to destructive interference at the cavity layer [Fig. 2(a)] and is essentially reflected by the dielectric multilayer with reflectivity near $100 \%$. For light at the resonant wavelength, its field intensity becomes maximum at the cavity layer due to constructive interference. Because of the TIR at the cavity layer-water interface, the light is reflected back with reflectivity also close to $100 \%$. Therefore, to have a resonant reflectance dip in the reflection spectrum, it is necessary to incorporate certain amount of absorption in the cavity layer. Thus, only when the light is at the resonant wavelength, can it experience the maximum absorption due to the enhanced field at the cavity layer, leading to a resonant reflectance dip in the reflectance spectrum.

The main distinction between the conventional Fabry-Pérot and the PC-TIR structure lies in the whereabouts of the energy of the nonreflected beam. The reflectance change of Fabry-Pérot ultrasound sensors is due to the change in intensity between 
reflected and transmitted beams caused by cavity thickness variation. In contrast, in the PC-TIR structure, no transmission is possible due to the TIR, and the reflectance change is induced by absorption inside the cavity, which is proportional to the interferometric field enhancement inside the cavity. One concern arises from the production of heat by light absorption inside the cavity, which may cause shifts of the operational wavelength or incident angle. However, deviation from expected operational condition was not observed, even in prolonged experiments. This is possibly because the cavity layer is in contact with the surrounding medium (i.e., water), which provided means of heat dissipation. There is an optimal absorption in the cavity layer to obtain a sharp and deep resonant reflectance dip. The measured FWHM of the dip is $1 \mathrm{~nm}$ [Fig. 2(b)], whereas the free spectral range is $53 \mathrm{THz}$, giving a finesse of 71 . Although the number of dielectric layers in our fabricated sample is relatively low (only five layers), due to large incident angle, the measured finesse is comparable to that of conventional étalon sensors, with more dielectric layers reported to date. ${ }^{6-9,16,20}$ This result demonstrates that a high finesse can be achieved for the open cavity in the PC-TIR configuration.

The transfer factor or responsitivity of a PC-TIR sensor depends on three factors: $(i)$ the conversion efficiency of the cavity-length change to the reflected light intensity change (optical sensitivity, $O_{s}$ ), (ii) the conversion efficiency of the ultrasonic pressure to the change in the resonance wavelength of the cavity (acoustic sensitivity, $A_{s}$ ), and (iii) the frequency response transfer function $P(k)$. For a PC-TIR sensor operating near the resonant condition, the maximum optical sensitivity is given by $^{18}$

$$
\left.O_{\mathrm{s}}\right|_{\max }=\left.\frac{\mathrm{d} I}{\mathrm{~d} \lambda}\right|_{\max }= \pm \frac{1.3 I_{0}\left(1-R_{\min }\right)}{\Delta \lambda},
$$

where $I_{0}$ is the incident probe laser intensity, $R_{\min }$ is the minimum reflectance at resonance, and $\Delta \lambda$ is the FWHM of the resonance dip.

The acoustic sensitivity represents the amount of strain produced by unit acoustic pressure (in micrometers per kilopascal). Because PMMA has a much lower Young's modulus compared to titania and silica used for the PC structure, the contribution from titania and silica to the acoustically induced strain is negligible compared to the contribution from PMMA. By neglecting the photoelastic and thermoelastic effects, the strain can be estimated by

$$
\text { strain }=\frac{\Delta d_{x}}{d_{x}}=-\frac{\Delta P}{E},
$$

where $\Delta P$ is the acoustic pressure and $E$ is the Young's modulus of the cavity material. From Eqs. (2) and (5), one obtains the acoustic sensitivity as

$$
A_{\mathrm{s}}=\frac{\partial \lambda_{\mathrm{d}}}{\partial P}=-\frac{4 n_{x} d_{x} \cos \theta_{x}}{(2 m+1-\alpha / \pi) E} .
$$

Assuming that the entire beam spot is subject to ultrasonic modulation, the calculated frequency dependence of the overall transfer factor $S$ is given by the product of $O_{s}, A_{s}$, and $|P(k)|$

$$
S=\frac{7.2 I_{0} n_{\mathrm{X}} d_{\mathrm{X}} \cos \theta_{\mathrm{X}}\left(1-R_{\min }\right)}{\Delta \lambda[2 m+1-(\alpha / \pi)] E}|P(k)| .
$$

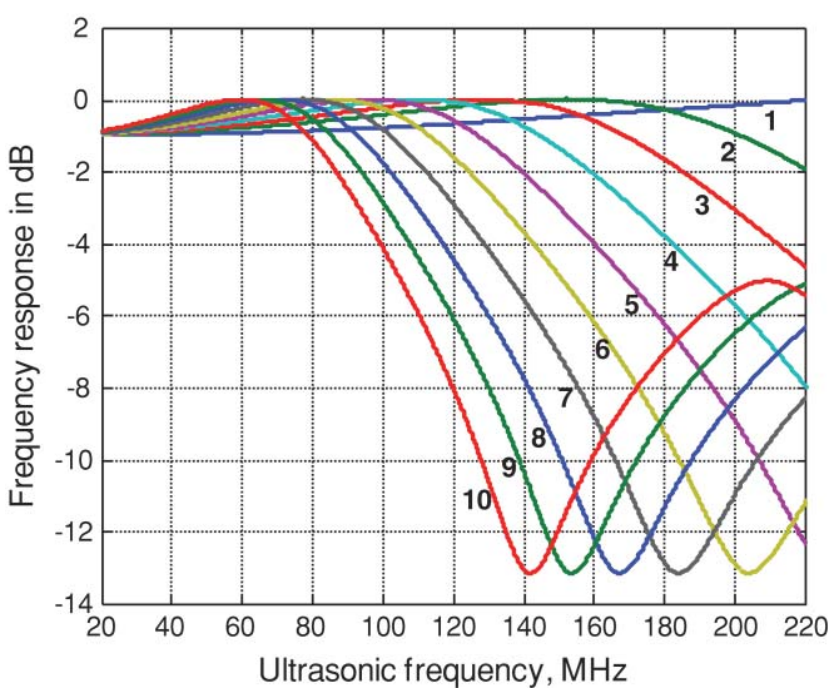

Fig. 3 Simulated frequency response of PC-TIR sensors with different cavity thickness. From line 1 to line 10, the cavity layer thicknesses are $2.31,3.76,4.48,5.21,5.93,6.66,7.38,8.11,8.83$, and $9.56 \mu \mathrm{m}$, respectively.

The frequency response transfer function $|P(k)|$ is a normalized, acoustic-frequency-dependent (or wavelength-dependent) function determined by the acoustic properties of the cavity layer, its backing material and its surrounding medium, and has the following form: ${ }^{13}$

$$
\begin{aligned}
& |P(k)|=\frac{T \sqrt{2}}{N k d_{x}} \\
& \quad \times\left\{\frac{\left(1+R_{1}^{2}\right)\left[1-\cos \left(k d_{x}\right)\right]+4 R_{1} \cos \left(k d_{x}\right) \sin ^{2}\left(k d_{x} / 2\right)}{1-2 R_{1} R_{2} \cos \left(2 k d_{x}\right)+\left(R_{1} R_{2}\right)^{2}}\right\}^{1 / 2},
\end{aligned}
$$

where $N$ is the normalization factor, $T$ is the pressure-amplitude transmission coefficient, $R_{l}$ and $R_{2}$ are the pressure-amplitude reflection coefficients at the lower and upper surfaces of the cavity, and $k=2 \pi / \lambda_{x}$ is the acoustic wave number, where $\lambda_{x}$ is the acoustic wavelength in the cavity. The calculated frequency response transfer function for different cavity thicknesses is shown in Fig. 3. This indicates that the frequency bandwidth increases with decreasing cavity thickness and a broadband response can be achieved with a reasonable cavity thickness.

To measure the transfer factor and frequency response of the PC-TIR sensor, we used a 2-mW He-Ne laser as the probe light with a wavelength of $632.8 \mathrm{~nm}$. The beam size was reduced to $\sim 0.5 \mathrm{~mm}$ diam using a pair of objective lenses forming a telescope. The PC-TIR sensor was placed on a glass prism, which was mounted on a rotational stage. The rotational stage enabled us to control the incident angle of the probe beam. A cylinder was placed around the sensor to hold water for the acoustic coupling. An $80-\mathrm{MHz}, 100-\mu \mathrm{m}$-focal-diam, single-element high-frequency piezoelectric ultrasound transducer (University of Southern California, Ultrasound Transducer Resource Center, Los Angeles, California) was driven by an ultrasound pulser (Olympus NDT pulser/receiver 5910PR) and positioned with its focus falling within the laser beam spot on the top surface of the sensor. The reflected probe laser beam was detected by 

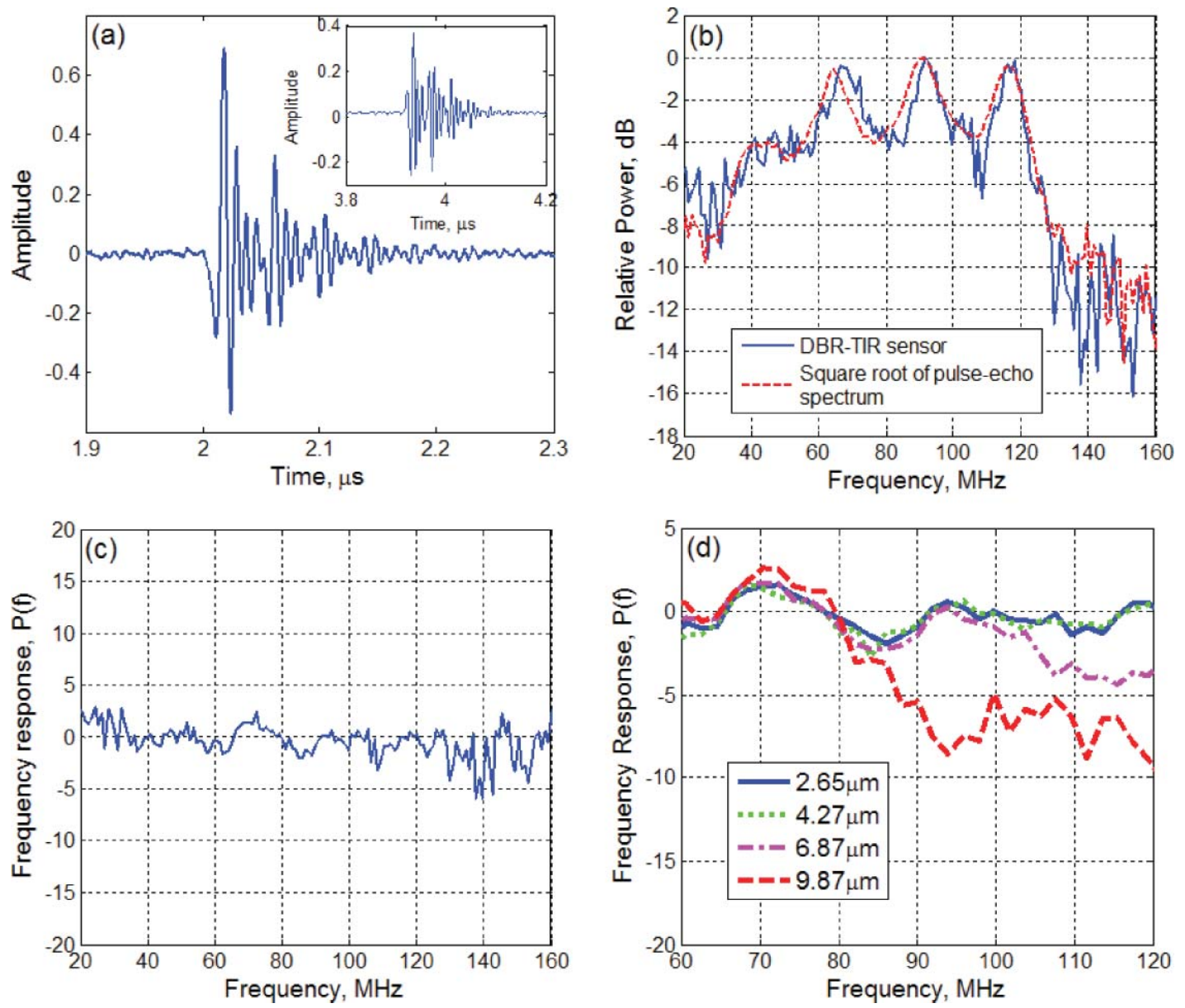

Fig. 4 Experimental testing of PC-TIR sensors in comparison with a piezoelectric transducer. (a) Ultrasonic signals from an 80-MHz transducer detected with a PC-TIR sensor having a PMMA cavity thickness of $2.65 \mu \mathrm{m}$ (inset: ultrasonic pulse-echo signal detected with the piezoelectric transducer); (b) frequency spectrum of the signals measured with the PC-TIR sensor and square-root of the pulse-echo spectrum; (c) frequency transfer function for the $2.65-\mu \mathrm{m}$ sensor obtained by dividing the PC-TIR spectrum by the pulse-echo spectrum; and (d) frequency spectra of the ultrasound signals measured with four different PC-TIR sensors having different cavity thicknesses.

a photodiode (Thorlabs PDA10A, 1-mm active area diameter) and was recorded using a digital oscilloscope (Agilent Infiniium 54830B)

Figure 4(a) shows the ultrasonic pulse signals detected by a PC-TIR sensor with a cavity thickness of $2.65 \mu \mathrm{m}$. A good SNR was obtained, indicating the sensitive response of the PC-TIR sensor to the ultrasonic signals. For comparison, the inset shows the ultrasonic pulse-echo (backscattered) signals received by the piezoelectric ultrasound transducer. The frequency spectrum of the detected pulse was determined by Fourier transform and found to be identical to that of the transducer within the calibration range of 10-80 MHz. Direct measurement of frequency response beyond $100 \mathrm{MHz}$ is difficult due to the unavailability of a well-calibrated ultrasound transducer and the lack of a calibration technique in this high frequency range. To make a rough estimate of the PC-TIR sensor frequency response, we assumed that the frequency response of the transducer in receiving mode is identical to that of transmission mode when subject to a broadband ultrasonic or electrical pulse. Also, assuming that the pulse is undistorted on reflection at the sensor surface, the normalized frequency spectrum of the pulse incident on the sensor surface is given by the square root of the (normalized) pulse-echo spectrum. This together with the spectrum measured by the PC-TIR sensor is shown in Fig. 4(b). A good agreement between the two spectra results in a relatively flat frequency response to at least $160 \mathrm{MHz}$, as shown in Fig. 4(c). Uncertainty arises at frequencies beyond $120 \mathrm{MHz}$ due to the low spectral power of the transducer.

We further investigated the relation between the cavity thickness and the frequency response. Frequency spectra of ultrasonic signals measured by four PC-TIR sensors with different cavity thicknesses are shown in Fig. 4(d). Two 3-dB cutoff frequencies are estimated to be $85 \mathrm{MHz}$ for the sensor with a cavity of $9.87 \mu \mathrm{m}$ and $110 \mathrm{MHz}$ for the sensor with a cavity of $6.87 \mu \mathrm{m}$. This dependence of frequency bandwidth on the cavity thickness is in good agreement with our simulation results (Fig. 3). The frequency response of the PC-TIR sensors with 2.65- and $4.27-\mu \mathrm{m}$ cavity layers have a bandwidth that is theoretically over $200 \mathrm{MHz}$ and is much broader than the testing ultrasonic source used in this measurement $(80 \mathrm{MHz})$.

The sensitivity of the PC-TIR sensor was measured by detecting the photodiode output when a calibrated ultrasonic source of known peak pressure was used. For the PC-TIR sensor with a cavity thickness of $4.27 \mu \mathrm{m}$, we obtained a sensitivity of $8 \mu \mathrm{W} / \mathrm{MPa}$. The NEP, given by detection noise divided by the sensor sensitivity, was found to be $36 \mathrm{kPa}$. Because this 
sensor has a broad frequency response [Fig. 4(d)], this sensitivity is expected to remain at this level at frequencies up to at least $120 \mathrm{MHz}$. Note that although the NEP is an order of magnitude larger than that of a similar-sized membrane hydrophone, ${ }^{15}$ it is mainly due to the electronic output noise of the photodiode used. In principle, the NEP of the PC-TIR sensor can be significantly reduced by using a low-noise photodiode and a higher power probe laser. Despite the high-detection-noise floor in our current detection system, our experimental results do indicate that our sensor has high sensitivity, which is determined by the optical structure and the materials used for fabricating the sensor. Although it is important to reduce the electronic noise, the high sensitivity demonstrated in this study is a critical step for obtaining an ultimately low NEP.

This study demonstrated the feasibility of constructing a high-finesse optical ultrasonic sensor using only half of a FabryPérot cavity structure. Despite a coarse proof-of-principle measurement, the sensor displayed a broad bandwidth of at least $160 \mathrm{MHz}$ with an NEP of $36 \mathrm{kPa}$, high sensitivity, and a potentially achievable low NEP if an improved detection method is employed. Substantial effort is necessary to fully evaluate the capability of the PC-TIR sensor at a high-frequency range. The PC-TIR sensor configuration addresses the trade-off problem in the other existing optical ultrasound sensors with a close cavity structure. This novel sensor provides total flexibility in cavity material selection to address various application requirements. We believe that it will find appeal in a wide range of biomedical applications that require sensitive, high-frequency ultrasonic imaging. For example, the high-sensitivity and broadband characteristics of the PC-TIR sensor developed in this study may lead to a significant improvement of the imaging quality of intravascular ultrasonic imaging for detecting vulnerable atherosclerotic plaques in the coronary artery. ${ }^{21}$ This novel optical ultrasound sensor may also be expected to have the potential to meet the increasing demand on the sensitivity and bandwidth required in photoacoustic imaging, a new imaging modality that has recently shown great promise for noninvasive characterization of internal structures and functions of soft tissues. ${ }^{22,23}$

\section{Acknowledgment}

Financial support by the U.S. National Institutes of Health (Grant No. R21EB008765 to J. Y. Ye) is acknowledged.

\section{References}

1. R. A. Smith, "Are hydrophones of diameter $0.5 \mathrm{~mm}$ small enough to characterise diagnostic ultrasound equipment?" Physics Med. Biol. 34, 1593-1607 (1989).

2. S. H. Lee, R. Maeda, and M. Esashi, "Microfabrication of thick and bulk PZT materials for piezoelectric actuator," Proc. SPIE 4936, 365372 (2002)

3. S. Ashkenazi, Y. Hou, T. Buma, and M. Odonnell, "Optoacoustic imaging using thin polymer etalon," Appl. Phys. Lett. 86, 134102 (2005).

4. P. Lum, M. Greenstein, C. Grossman, and T. L. Szabo, "Highfrequency membrane hydrophone," IEEE Trans. Ultrason. Ferroelectr. Freq. Control 43, 536-544 (1996).
5. V. Wilkens and W. Molkenstruck, "Broadband PVDF membrane hydrophone for comparisons of hydrophone calibration methods up to $140 \mathrm{MHz}$," IEEE Trans. Ultrason. Ferroelectr. Freq. Control 54, 17841791 (2007).

6. E. Z. Y. Zhang and P. C. Beard, "Ultrahigh-sensitivity wideband Fabry-Perot ultrasound sensors as an alternative to piezoelectric PVDF transducers for biomedical photoacoustic detection," Proc. SPIE 5320, 222-229 (2004).

7. V. Wilkens, "Characterization of an optical multilayer hydrophone with constant frequency response in the range from 1 to $75 \mathrm{MHz}$," J. Acoust. Soc. Am. 113, 1431-1438 (2003).

8. E. Zhang, J. Laufer, and P. Beard, "Backward-mode multiwavelength photoacoustic scanner using a planar Fabry-Perot polymer film ultrasound sensor for high-resolution three-dimensional imaging of biological tissues," Appl. Opt. 47, 561-577 (2008).

9. E. Zhang and P. Beard, "Broadband ultrasound field mapping system using a wavelength tuned, optically scanned focused laser beam to address a Fabry Perot polymer film sensor," IEEE Trans. Ultrason. Ferroelectr. Freq. Control 53, 1330-1338 (2006).

10. P. C. Beard and T. N. Mills, "Miniature optical fibre ultrasonic hydrophone using a Fabry-Perot polymer film interferometer," Electron. Lett. 33, 801-803 (1997).

11. J. P. Monchalin, "Optical detection of ultrasound at a distance using a confocal Fabry-Perot interferometer," Appl. Phys. Lett. 47, 14-16 (1985).

12. V. Wilkens, "Characterization of an optical multilayer hydrophone for use as broadband ultrasound reference receiver-comparison with PVDF membrane hydrophones," in Proc. IEEE -Ultrasonics Symp., Vol. 771, pp. 773-776 (2002)

13. P. C. Beard, F. Perennes, and T. N. Mills, "Transduction mechanisms of the Fabry-Perot polymer -film sensing concept for wideband ultrasound detection," IEEE Trans. Ultrason Ferroelectr. Freq. Control 46, 1575 1582 (1999).

14. B. T. Cox and P. C. Beard, "The frequency-dependent directivity of a planar Fabry-Perot polymer film ultrasound sensor," IEEE Trans. Ultrason Ferroelectr. Freq. Control 54, 394-404 (2007).

15. P. C. Beard, A. Hurrell, E. Van Den Elzen, and T. N. Mills, "Comparison of a miniature, ultrasonic, optical fibre hydrophone with PVDF hydrophone technology," in Proc. IEEE Ultrasonics Symp., Vol. 1882, pp. 1881-1884 (1998).

16. W. Weise, V. Wilkens, and C. Koch, "Frequency response of fiber-optic multilayer hydrophones: experimental investigation and finite element simulation," IEEE Trans. Ultrason Ferroelectr. Freq. Contol. 49, 937945 (2002)

17. C. Y. Shih, T. A. Harder, and Y. C. Tai, "Yield strength of thin-film parylene-C," Microsyst. Technol. 10, 407-411 (2004).

18. Y. Guo, C. Divin, A. Myc, F. L. Terry Jr., J. R. Baker Jr., T. B. Norris, and J. Y. Ye, "Sensitive molecular binding assay using aphotonic crystal structure in total internalreflection," Opt. Express 16, 11741-11749 (2008).

19. J. Y. Ye and M. Ishikawa, "Enhancing fluorescence detection with a photonic crystal structure in a total-internal-reflection configuration," Opt. Lett. 33, 1729-1731 (2008).

20. B. T. Cox, E. Z. Zhang, J. G. Laufer, and P. C. Beard, "Fabry Perot polymer film fibre-optic hydrophones and arrays for ultrasound field characterisation," J. Phys. Conf. Ser. 1, 32-37 (2004).

21. A. Nair, B. D. Kuban, E. M. Tuzcu, P. Schoenhagen, S. E. Nissen, and D. G. Vince, "Coronary plaque classification with intravascular ultrasound radiofrequency data analysis," Circulation 106, 2200-2206 (2002).

22. S. Sethuraman, S. R. Aglyamov, J. H. Amirian, R. W. Smalling, and S. Y. Emelianov, "Intravascular photoacoustic imaging using an IVUS imaging catheter," IEEE Trans. Ultrason. Ferroelectr. Freq. Contr. 54, 978-986 (2007).

23. P. M. Henrichs, J. W. Meador, J. M. Fuqua, and A. A. Oraevsky, "Atherosclerotic plaque characterization with optoacoustic imaging," Proc. SPIE 5697, 217-223 (2005) 